\title{
Combinación de métodos potenciales y percepción remota para el análisis geológico-estructural de la caldera panales, Hidalgo, México
}

\author{
J.A. Reyes Moreno ${ }^{1}$, F.Y. Pérez Corona ${ }^{1 *}$, I. Árcega Santillán ${ }^{1}$, L.E. Ortíz Hernández \\ ${ }^{1}$ Universidad Autónoma del Estado de Hidalgo, Instituto de Ciencias Básicas e Ingeniería, Área Académica \\ de Ciencias de la Tierra, Ingeniería en Geología Ambiental, Ciudad del Conocimiento Kilómetro 4.5 \\ carretera Pachuca - Tulancingo, Colonia Carboneras, Mineral de la Reforma, Hidalgo, 42184, México. \\ re284130@uaeh.edu.mx, ingrid_arcega@uaeh.edu.mx, leoh44@hotmail.com.
}

*Autor de correspondencia: fred_perez@uaeh.edu.mx

\section{RESUMEN}

La zona de estudio se ubica entre las provincias fisiográficas de la sierra madre oriental y la faja volcánica transmexicana. Geológicamente, corresponde a la plataforma vallesSan Luis Potosí, formada durante el cretácico en la porción este y centro de México. De manera específica, el área de estudio se sitúa en la caldera panales (CP) con un diámetro aproximado de 9 kilómetros, exponiendo diversos domos, diques dacíticos y un intrusivo de composición diorítica que provocó metamorfismo de contacto en la caliza encajonante. El objetivo del presente trabajo es obtener un modelo geológico-geofísico del subsuelo de la CP.

Para el estudio e interpretación del subsuelo se combinaron métodos potenciales (gravimetría y magnetometría) e imágenes Sentinel2, a los cuales se les aplicaron diferentes algoritmos matemáticos. El análisis indica que existe una correlación entre los lineamientos obtenidos de los métodos potenciales y la percepción remota con las diferentes fallas, fracturas y contactos geológicos, de igual manera con la modelación 2D se obtuvo la geometría relativa de la CP. Los resultados obtenidos corroboran que la estructura semicircular de Panales corresponde a una caldera, así como su correlación con los diferentes eventos tectónicos y volcánicos.

Palabras Clave: Caldera Panales, métodos potenciales, percepción remota.

\section{INTRODUCCIÓN}

El estado de Hidalgo se encuentra situado en la parte central de México, es un estado el cual no es caracterizado por alta actividad sísmica debido al hecho de su lejanía a las zonas costeras pacíficas. Sin embargo, los principales rasgos de tectónica activa en Hidalgo, se concentran en la faja volcánica transmexicana, ya que es una provincia volcánica joven con edades de plioceno-cuaternario, manifestándose vulcanismo y tectónica activa en la actualidad [11].

El área de estudio se ubica en la CP, situada al SW de Ixmiquilpan y al sur del poblado de panales, estructuralmente se encuentra dentro de tres principales sistemas de fallas (el semigraben de Aljibes, el graben del Mezquital y el semigraben de Tlaxcoapan) que pudieron directa o indirectamente contribuir en su formación [11].

Existen diversas aplicaciones de los sistemas de información geográfica (SIG's) y métodos geofísicos en el estudio de calderas volcánicas, las cuales permiten realizar un 
modelado morfométrico. Mediante la combinación de métodos potenciales, cálculos matemáticos y percepción remota además se pueden obtener mediciones precisas de parámetros horizontales como: diámetro y longitud o verticales como: altura y profundidad, las cuales son el principal objeto de estudio en el presente trabajo.

\section{MARCO TEÓRICO}

Una caldera se define como una depresión circular de más de 1 km de diámetro formada por el colapso del terreno y que es posterior a una erupción volcánica. Las características morfológicas relevantes de una caldera son una estructura circular delimitada por fracturas anilladas, la ocurrencia de domos en su periferia y hacia su centro (domos resurgentes), otros elementos característicos de las calderas pueden incluir el relleno intracaldera y la cámara magmática subyacente. Por su naturaleza, una caldera constituye una estructura con marcada inestabilidad de los bloques de roca hacia la periferia [6]. En una imagen de satélite se pueden observar varios objetos de diferentes tamaños y formas, algunos de ellos se identifican correctamente mientras que otros no, dependiendo de las percepciones individuales y de la experiencia del interprete [5]. Esto nos permite ver la zona de estudio completa y analizar si existe una relación directa de una estructura con otra. Esta técnica fue utilizada en este estudio para correlacionar la forma de la caldera con la geología de la zona. La magnetometría mide a través de líneas planificadas en superficie la intensidad del campo magnético total (CMT), conteniendo una componente regional debido al campo magnético terrestre producido por el núcleo (dipolo principal) y una componente residual debido al efecto de los minerales magnéticos en las rocas presentes en la corteza Terrestre.

Los mapas magnéticos permiten interpretaciones cualitativas de estructuras geológicas y definición de unidades en base a la cantidad de minerales magnéticos en las rocas. Una siguiente etapa corresponde a la interpretación cuantitativa mediante el análisis numérico de los datos (modelamiento e inversión) la cual permite determinar la ubicación, forma y profundidad de los cuerpos magnetizados [2]. La gravimetría consiste en la medición del campo de gravedad. Se suele emplear cuando el objeto de estudio es el campo de gravedad o las variaciones de densidad responsables de su variación. Esto consiste en la medición de la aceleración de gravedad sobre un terreno con el fin de detectar las variaciones de densidades en las unidades geológicas del subsuelo [3].

\subsection{Geología}

La geología de la CP está compuesta por diferentes unidades geológicas (Figura 1), presentando brechas dacíticas en los bordes de los cerros Dianxe, La Cruz, Xintza y Dexitzo del Terciario Superior, a las cuales les sobreyace una secuencia de andesita parcial o totalmente oxidada, de la misma edad [13].

En la parte central de la CP se tiene un conjunto de basaltos compuestos por olivino y clinopiroxeno, al oeste calizas arrecifales de la Formación El Abra (Cretácico Inferior), calizas con lutita de la Formación Soyatal (Cretácico Superior) y al sur-oeste se encuentran lutitas intercaladas con arenisca de la Formación Mezcala (Cretácico superior) [7]. Rodeando a estas unidades se encuentra un conglomerado de areniscas perteneciente al Neógeno. 
La CP se define como una caldera volcánica subsidente con indicios de actividad resurgente. Asimismo, se han propuesto tres episodios caldericos para la CP; 1) Evento pre-caldera el cual es una extrusión de lavas andesítica y basálticas presentes en el anillo de caldera; 2) Evento generador de caldera y colapso, el cual comprende una explosión ignimbrítica y colpa de la parte superior adyacente de la cámara magmática y 3) Evento post-caldera resurgente, el cual es inyección de diques y formación de domos al centro de la estructura [11].

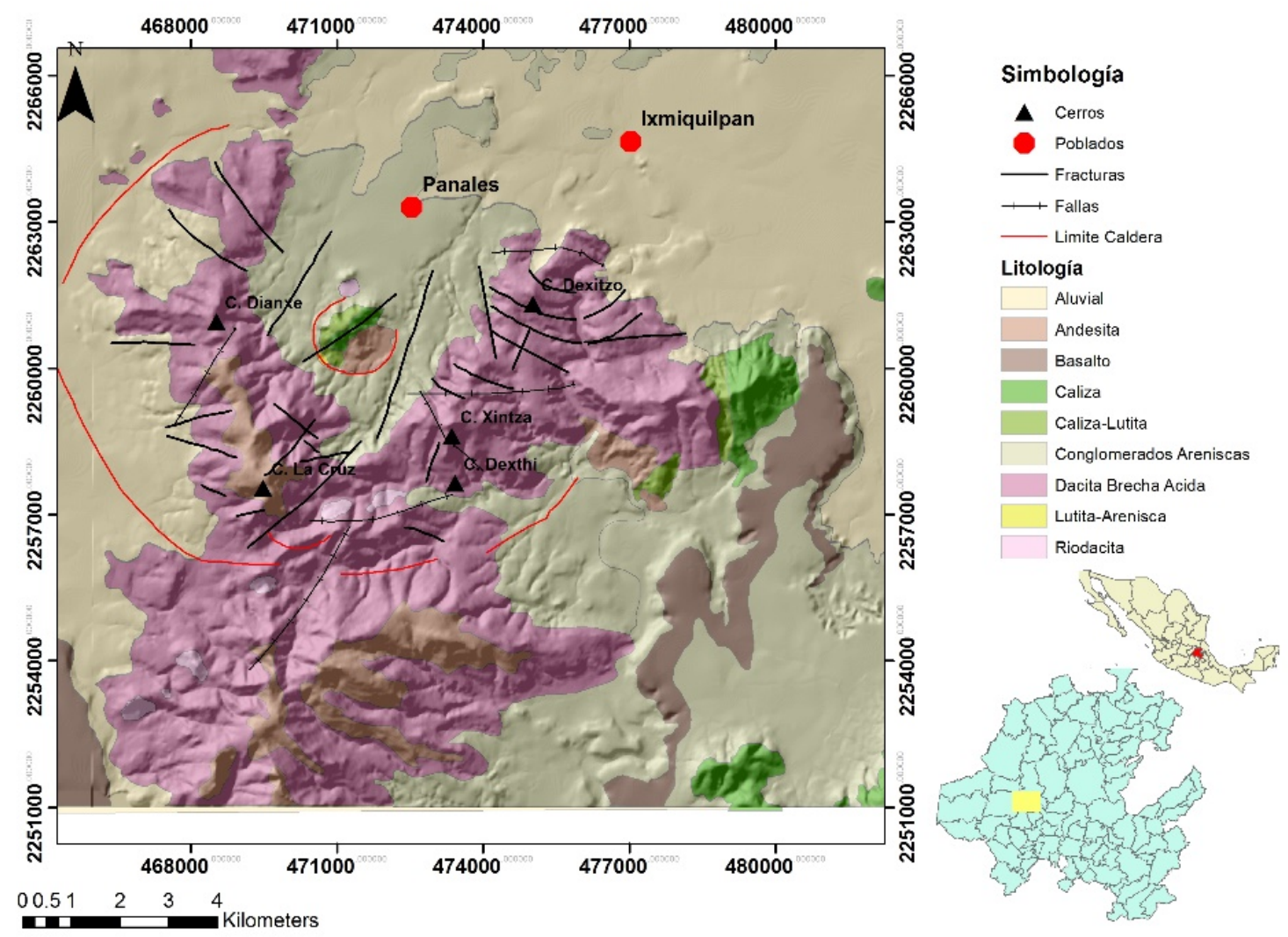

Figura 1. Plano geológico simplificado de la Caldera Panales

\section{PARTE EXPERIMENTAL}

\subsection{Percepción Remota}

El análisis de percepción remota consistió en correlacionar la información de la carta Ixmiquilpan [13], el contínuo de elevación mexicano (CEM) de INEGI e imágenes Sentinel 2.

Se combinaron las bandas 12, 4 y 2 del satélite Sentinel 2 para identificar elementos geológicos [12], lo anterior permitió realzar los contrastes en el área de estudio. Existe un contraste claro entre la morfología visible de la CP, fallas y fracturas que se localizan en la zona. La caldera se encuentra delimitada por los cerros; Dianxe, La Cruz, Dexthi, Xintza y Dexitzo, señalados por las líneas rojas en la Figura 2. 


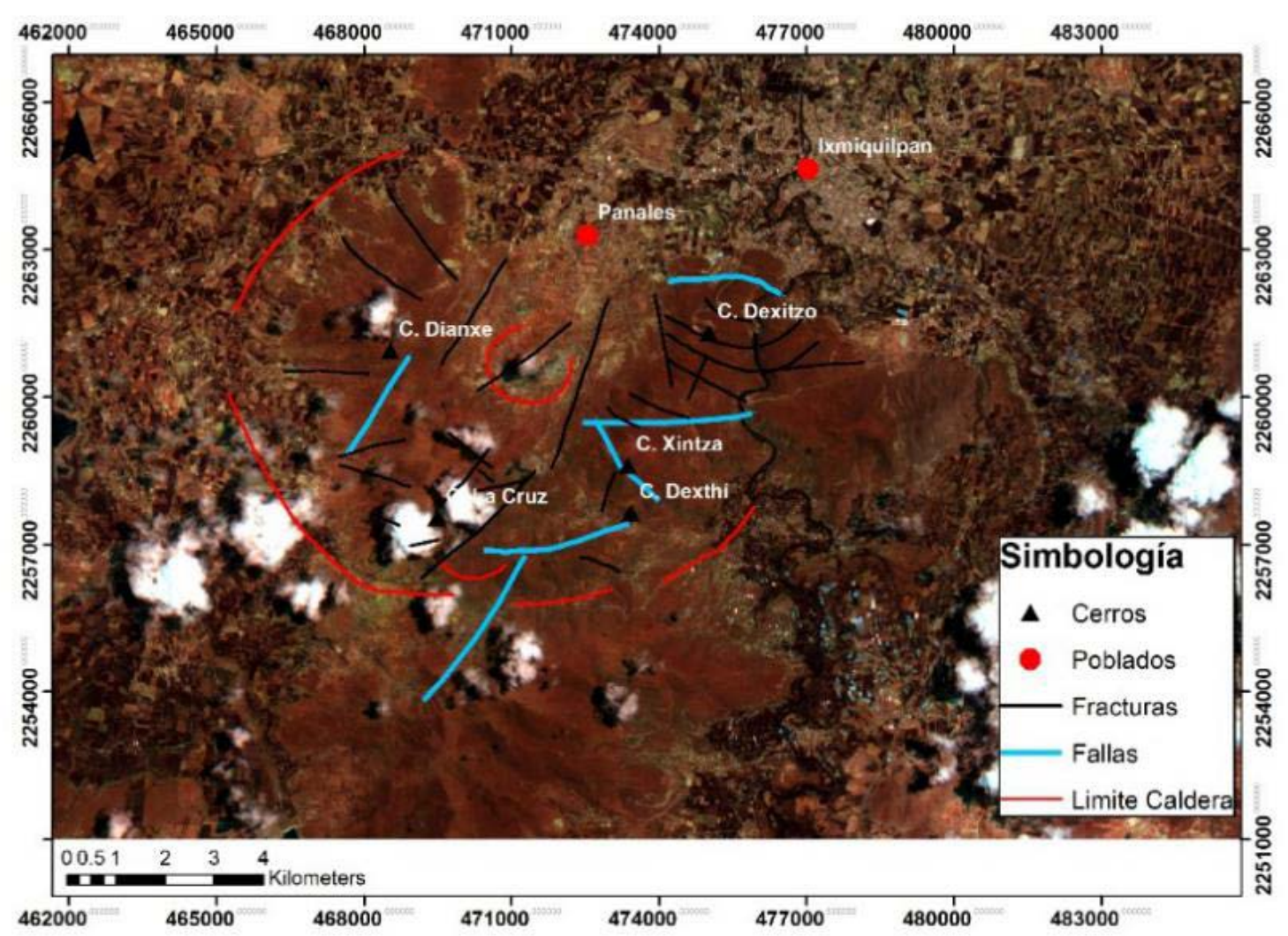

Figura 2. Combinación de las bandas 12,4 y 2 de la imagen satelital Sentinel 2

\subsection{Métodos potenciales}

Magnetometría: Los datos de magnetometría aérea (CMT) utilizados fueron obtenidos por el Servicio Geológico Mexicano, restándoles el Campo Geomagnético Internacional de Referencia (IGRF por sus siglas en inglés) obteniendo la Anomalía Magnética (AM), para posteriormente extraer el Campo Magnético Residual del regional, al que se le aplicó el algoritmo de Reducción al Polo [14] (Figuras 3B) para ubicar las anomalías magnéticas directamente sobre las fuentes que las causan.

Gravimetría: La anomalía de Bouguer de los datos de gravimetría satelital se obtuvo a través del Bureau Gravimétrique International (BGI), la cual se deriva de valores satelitales y del modelo gravitacional terrestre EGM2008 elaborado por la NGA (National Geospatial-Intelligence Agency), se corrigieron utilizando el código FA2BOUG [9]. La corrección topográfica se aplicó considerando el CEM de INEGI, resolución 15 ×15m de por pixel. Al conjunto de datos se les realizó la corrección por terreno utilizando el método de Hammer [5] obteniendo la Anomalía de Bouguer Completa (ABC) y subsecuentemente se separó el residual (Figura $3 A$ ) del regional aplicando un filtro polinomial de segundo grado [8]. 

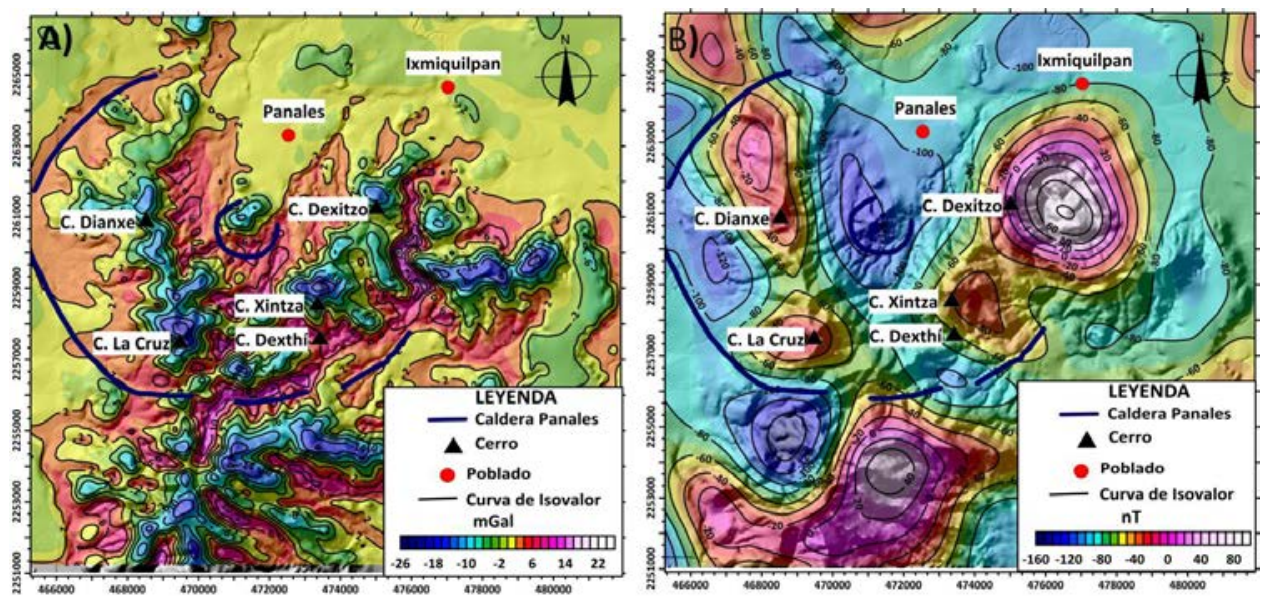

Figura 3. Planos de los métodos potenciales, A) Anomalía Residual de Bouguer de la Gravimetría Satelital, B) Campo Magnético Residual Reducido al Polo de la Magnetometría Aérea.

Ángulo de inclinación (Tilt derivative): El ángulo de inclinación (Al) iguala las amplitudes de la señal, y los picos de los datos transformados se sitúan sobre el centro del cuerpo que los causa, es usada a menudo para detectar los bordes en contactos geológicos, que pueden representar fracturas y/o fallas geológicas [4]. El Al es una herramienta muy útil para mapear tanto fuentes someras como fuentes profundas, se define por el arco tangente de la división de la Primera Derivada en la Vertical (PDV) entre la Derivada Horizontal Total (DHT).

$$
\mathrm{Al}=\arctan (\mathrm{PDV} / \mathrm{DHT})
$$

Indicando el vector resultante de la combinación de las primeras derivadas horizontales en las direcciones $x$ y $y$, acentuando los cambios laterales abruptos de las propiedades físicas de las estructuras presentes en el subsuelo [1]. La principal ventaja del filtro Al es que el valor de contorno igual a cero está cercano o se localiza sobre las fallas, fracturas, contactos o algún otro lineamiento geológico. En el área de estudio se aplicó este filtro a los datos de gravimetría satelital y magnetometría aérea (Figura 4A y 4B), pudiéndose caracterizar y delimitar diferentes lineamientos del área de estudio.
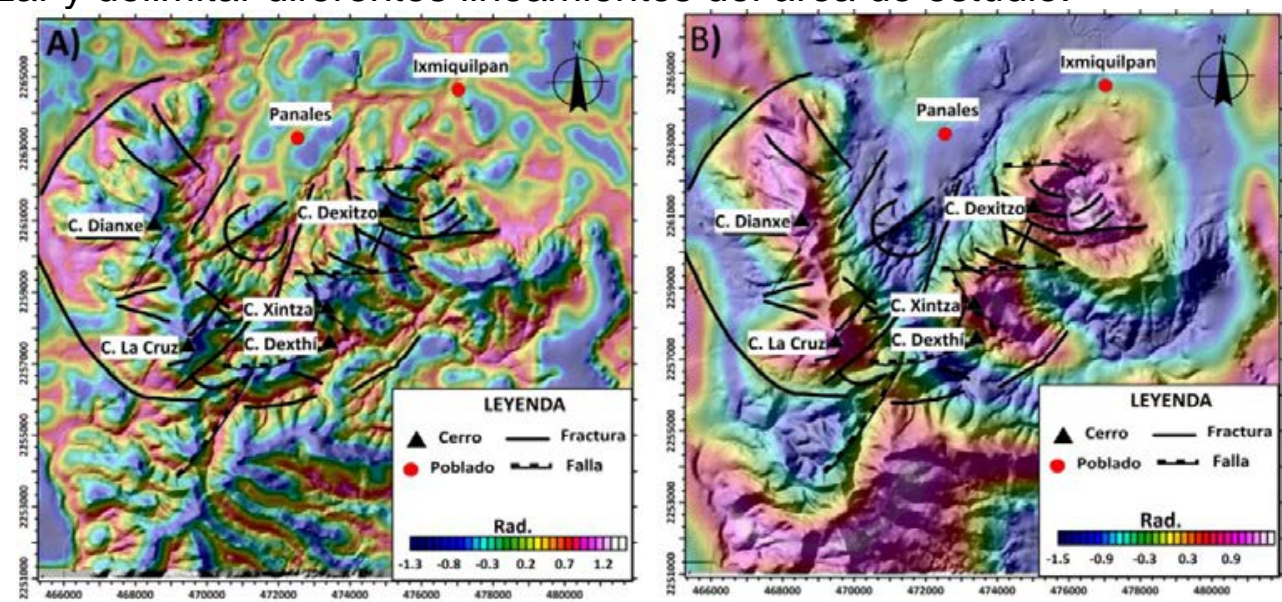

Figura 4. Planos del Ángulo de Inclinación, A) Anomalía Residual de Bouguer de la Gravimetría

Satelital, B) Campo Magnético Residual Reducido al Polo de la Magnetometría Aérea. 


\section{RESULTADOS}

Para la caracterización de los lineamientos se realizaron diversos mapas en los que se combinaron la topografía, las bandas 12, 4 y 2 del satélite Sentinel 2 y se aplicó el algoritmo de Al, permitiendo obtener la correlación que existe de las estructuras circulares con los diferentes conjuntos de datos. A partir de la configuración de los datos y aplicación de algoritmos matemáticos se pudieron interpretar los lineamientos principales (Figura 4) en la CP característicos de diversas estructuras geológicas, apreciándose claramente en el mapa la presencia de diversas fallas, fracturas y algunos lineamientos circulares que pueden estar asociados a estructuras de carácter regional.

El mapa de Al muestra que las diferentes anomalías pueden estar asociadas a fallas de carácter regional relacionadas con el origen y evolución de la estructura circular de la CP. En la figura 2 se aprecia una relación estrecha entre las formaciones geológicas como un río asociado al sistema de fallas y fracturas presentes en la CP. Además se observa la delimitación de forma circular de la CP.

\section{CONCLUSIONES}

El uso de imágenes satelitales, mediante sistemas de información geográfica, delimitaron los principales rasgos morfológicos, permitiendo establecer los límites de la Caldera de Panales. La combinación de bandas 12, 4 y 2 del satélite Sentinel 2 ayudó a discriminar de manera visual y extraer la litología de interés para este trabajo. La integración de diferentes datos potenciales junto con la aplicación de la percepción remota y el algoritmo matemático del ángulo de inclinación, caracterizaron de manera eficiente los lineamientos geofísicos-estructurales y la identificación de la caldera panales permitiendo así hacer un análisis geológico-estructural de la zona. De acuerdo a la asociación de las anomalías 2D obtenidas de los métodos potenciales, es recomendable implementar algún otro método geofísico como el magnetotelúrico y/o sísmica los cuales permitan dar una mayor resolución del subsuelo para realizar un modelo conceptual 3D, con la finalidad de comprender de mejor manera el origen y evolución de la caldera panales.

\section{BIBLIOGRAFÍA}

[1] B. Verduzco, J. Derek, C. Green, C. Mackenzie. The Leading Edge. 23 (2004) 116119.

[2] Geofísica de Avanzada by Geodatos, Recuperado el 2 de julio de 2019 de http://www.geodatos.cl/magnetometria.php.

[3] Geofísica de Avanzada by Geodatos, Recuperado el 2 de julio de 2019 de http://www.geodatos.cl/gravimetria.php.

[4] H.G. Miller, V. Singh. Journal of Applied Geophysics. 32 (1994) 213-217.

[5] H.R. Burger. (1992) Exploration geophysics of the shallow subsurface 2da. Ed., Prentice Hall.

[6] A.R. Hernández García. (2018) Análisis morfo-estructural para determinar la posible presencia de una caldera en el campo volcánico Río Santa María - El Órgano mediante el uso de técnicas geomáticas. Tesis de Maestría UASLP, San Luis Potosí, México.

[7] L. Pérez Aguilar. (2012) Geología del cerro siete minas, Ixmiquilpan, Hidalgo, México: Implicaciones en el ahogamiento de la porción sureste de la plataforma Valles - San 
Luis Potosí (plataforma de Actopan: Cretácico) y la evolución cenozoica regional. Tesis de Licenciatura UAEH, Pachuca de Soto, Estado de Hidalgo, México.

[8] M. Beiki, L. Bastani, B. Mehrdad, L.B. Pedersen. Geophysics 75 (2010) I37-I49.

[9] N.K. Pavlis, S.A. Holmes, S.C. Kenyon, J.K. Factor. J. Geophys. Res. (2012) 117 B04406.

[10] National Oceanic and Atmospheric Administration NOAA (2010) Recuperado el 25 de abril de 2019 de http://www.ngdc.noaa.gov/IAGA/vmod/igrf.html.

[11] S. Barrera Guerrero. (2009) Caracterización Geológica de la estructura semicircular Panales, Ixmiquilpan. Tesis de Licenciatura UAEH, Pachuca de Soto, Estado de Hidalgo, México.

[12] Sentinel Hub by Sinergise. Recuperado el 3 de marzo de 2019 de https://sentinelhub.com.

[13] Servicio Geológico Mexicano (SGM) (1995), Carta Geológico-Minero Ixmiquilpan F14-C79 Hidalgo: escala 1:50 000, Secretaria de Economía.

[14] V. Baranov, H. Naudy. Geophysics 29 (1964) 67-79. 